\title{
La radio en disputa: el concepto de campo en el fenómeno radiofónico en México*
}

\author{
Radio in dispute: The concept of the field \\ in the radio phenomenon in Mexico
}

\author{
ANAYURI GÜEMES CRUZ**
}

\begin{abstract}
This work is a study of the radio phenomenon that utilizes the conceptual tool of power field. It starts from a documentary review that takes up Bourdieu and Latour to analyze radio as a network of power relations historically constituted and in constant struggle. Consequently, this critical approach reveals the inequitable conditions of the radio field in Mexico and in its subfields. Technology as a reconfiguration factor of the radio field is a line to follow in future studies and reveals how important it is to generate more current anthropological studies on radio diversity in Mexico, which implies closing the gap between doing radio and thinking about radio.
\end{abstract}

Key words: broadcasting, power, agents, fight, state

\section{Introducción}

$\mathrm{E}$ 1 ámbito de la radio pública en México es un nodo colmado de heterogeneidad debido a sus contenidos y distintas ofertas culturales sonoras. Si bien hay cierto conocimiento sobre la radio, existe poca información cualitativa sobre lo que sucede con las instituciones radiofónicas concretas, y aún menos sobre sus públicos. Si se genera mayor investigación sobre las instituciones radiofónicas y sus públicos será posible sensibilizar acerca de la importancia de pensar y trabajar la radio a partir de sus transformaciones actuales para el fortalecimiento de la oferta radiofónica, frente a la casi omnipresente oferta de la radio comercial en México. Para lograr esta encomienda y analizar el fenómeno disponemos de una herramienta conceptual proporcionada por

\footnotetext{
* Artículo recibido el 03/03/20 y aceptado el 13/05/20.

** Benemérita Universidad Autónoma de Puebla.4 Sur núm. 104, Centro Histórico, 72000, Puebla, Pue.<anayuri.guemes@ correo.buap.mx>. ORCID: https://orcid.org/0000-0001-7520-3695
}

\begin{abstract}
Resumen
Este trabajo es un estudio del fenómeno radiofónico realizado con la herramienta conceptual de campo de poder. Parte de una revisión documental que recupera a Bourdieu y Latour para analizar la radio como un entramado de relaciones de poder históricamente constituido y en constante pugna. Este enfoque crítico revela las condiciones inequitativas del campo radiofónico y sus subcampos en México. La tecnología como factor de reconfiguración del campo radiofónico es una línea a seguir en futuros estudios y pone de manifiesto lo importante que es la generación de más estudios antropológicos sobre la diversidad radiofónica en México, lo que implica cerrar la brecha entre hacer radio y pensar la radio.
\end{abstract}

Palabras clave: radiodifusión, poder, agentes, lucha, Estado 
Pierre Bourdieu y su categoría de campo. Se abordará la conformación del campo radiofónico, a fin de saber cómo se ha configurado este espacio en pugna, las fuerzas que lo componen, así como los grupos e intereses que han estado involucrados. Después, nos acercaremos al campo radiofónico en México. ${ }^{1}$ Adicionalmente, expondremos los subcampos que expresan la práctica radiofónica más conocida en México, que hasta la fecha siguen manteniendo gran influencia, simbólica y materialmente, sobre el espacio radiofónico general. Y cerraremos con una reflexión sobre la vigencia de Bourdieu para el abordaje y problematización del fenómeno radiofónico en el país.

\section{Bourdieu y los campos}

Para generar una mirada que permita visibilizar y profundizar las tensiones y luchas dentro del ámbito radiofónico desde diversos ángulos considero necesario retomar a Pierre Bourdieu (2002; 2012), a partir de su concepto de campo, el cual permite explicar las asimetrías y la heterogeneidad del mundo radiofónico en México. Este concepto de campo establece un perímetro que nos sitúa en un determinado conjunto de relaciones de poder históricamente construidas y en constante movimiento por diferentes agentes, ${ }^{2}$ desde donde se puede entender a las distintas radios en México como el resultado de una trama relacional que sitúa y vincula a los agentes en relación con su práctica radiofónica y el poder del Estado.

Bourdieu (2002: 4) define que un campo: "no es un espacio neutro de relaciones interindividuales, sino que está estructurado como un sistema de relaciones en competencia y conflicto entre grupos y situaciones en posiciones diversas, como un sistema de posiciones sociales a las que están asociadas posiciones intelectuales y artísticas". En otras palabras, el campo es un espacio donde las posiciones sociales que ocupan los distintos agentes están en conflicto y se compite por su ocupación. Como un sistema de relaciones sociales, el campo está marcado por las disputas que los agentes sociales generan. En cuanto categoría, el campo de poder es adecuado para analizar la lógica del ámbito radiofónico.

Bourdieu (2002) sostiene que ningún campo puede entenderse de forma aislada, ya que se encuentra compuesto por un entramado de relaciones sociales a manera de un campo magnético con líneas de fuerza, dinámicas y cambiantes, entre los agentes que lo constituyen. La pertenencia y participación de los agentes dentro del campo se debe a la posición ocupada en él. Para efectos de este artículo, considerar el ámbito radiofónico desde la perspectiva del campo de Bourdieu (2002) permite identificar las relaciones y posiciones que lo constituyen, así como los nexos/tensiones de los agentes sociales que ocupan o establecen pugnas determinadas por dichas posiciones. Es esencial entender que el campo es histórico, sus posiciones, agentes y tensiones se han estructurado; de ahí que, para entender la lógica imperante de cada campo y los subcampos que lo conforman, sea imprescindible conocer esa historia. Para Bourdieu,

La estructura del campo es un estado de la relación de fuerzas entre los agentes o las instituciones que intervienen en la lucha o [...] de la distribución del capital específico que ha sido acumulado durante luchas anteriores y que orienta las estrategias ulteriores. Esta misma estructura, que se encuentra en la base de las estrategias dirigidas a transformarla, siempre está en juego: las luchas que ocurren en el campo ponen en acción al monopolio de la violencia legítima (autoridad específica) que es característico del campo considerado, esto es, en definitiva, la conservación o subversión de la estructura de la distribución del capital específico [Bourdieu, 2002: 120].

Es posible notar entonces que, dentro del campo, el poder deberá ser entendido como un proceso, como una energía que circula constantemente, no como una reserva que contiene una explicación, puesto que "[e]l poder y la dominación tienen que ser producidos, inventados, compuestos. Las asimetrías efectivamente existen, pero ¿de dónde vienen y de qué están hechas?" (Latour, 2008: 96) y ¿qué tipo de implicaciones tienen en lo social?, éstos son aspectos en los que debe pro-

1 Es relevante mencionar que este artículo es producto de la tesis doctoral "El campo radiofónico universitario, sus agentes y sus públicos: caso de Radio BUAP”, iniciada en 2012 y defendida en 2017 (Güemes Cruz, 2017); no obstante, se continuó con el trabajo de investigación, y el trabajo de campo comprende los años 2014-2019, el cual incluyó entrevistas informales, formales y a profundidad. Estas últimas no forman parte de este artículo, pues se pretende dar mayor peso a la revisión teórica e historiográfica mediante el análisis de la categoría de campo. Se espera desarrollar en siguientes artículos el trabajo de campo -etnografía y entrevistas diversas- del mencionado estudio.

2 Agente será entendido como el individuo o conjunto de éstos, así como instituciones u organizaciones que ocupen una posición dentro del campo, es decir, dentro del entramado de fuerzas de poder, por lo que no es posible entender un agente de manera aislada, como tampoco es posible definir al campo sin los agentes que lo ocupan, estará constituido por las posiciones y fuerzas que en el campo se establecen. El agente "está determinado por su pertenencia al campo [...] por lo que su autoridad no puede definirse independientemente de su posición en él” (Bourdieu, 2002: 9-10). 
fundizarse dentro del campo radiofónico, lo cual dará la pauta para develar los subcampos que lo componen.

Bruno Latour ha sido un gran guía para caminar dentro de la temática radiofónica contemporánea, en especial a partir de las últimas transformaciones sociales, pues existe un constante movimiento y podría ser confuso entender lo radiofónico si se observa de manera superficial. Este autor propone una forma distinta de comprender lo "social": a partir de vincular elementos heterogéneos mediante un rastreo de asociaciones (Latour, 2008).

Para Latour, lo social no es una amalgama de individuos en armonía, sino un campo de disputas cuya característica es que siempre está en movimientoy, por ende, se va reconfigurando. Es decir, las sociedades se encuentran en transformación, lo que demanda un reordenamiento constante de lo que sucede a nuestro alrededor $\mathrm{y}$, por consecuencia, debe haber también un reordenamiento en las formas de comprenderlas. Asimismo, para los agentes, este constante movimiento y reconfiguración de las sociedades altera su sentido de pertenencia, en palabras de Latour "El sentido de pertenencia ha entrado en crisis. Pero para registrar esta sensación de crisis y seguir estas nuevas conexiones es necesario idear una nueva noción de lo "social" es por ello necesario entender lo social como un movimiento muy peculiar de reasociación y reensamblado" (Latour, 2008: 20).

Lo social y la noción de campo de Bourdieu coinciden porque se refleja la visión de conflicto, disputa y movimiento. Para examinar el fenómeno radiofónico desde un enfoque social hace falta renovar las herramientas teóricas y, de este modo, idear una noción de lo social distinta, no como un conglomerado fijo de condiciones sociales, sino como uno dinámico, fugaz y en incesante movimiento. Aplicado a lo radiofónico, lo anterior implica entender la dinámica de la radio a partir de conocer las asociaciones establecidas a través del tiempo entre sujetos, agentes, instituciones y posiciones, así como relaciones, luchas y tensiones (lo cual involucra tanto a sujetos vinculados al quehacer radiofónico como a instituciones radiofónicas y a todo lo que conlleva su estructura, así como a las tensiones dentro y fuera de dichas instituciones), además de su relación frente al poder dominante del Estado en relación con el uso legítimo de las ondas hertzianas en México. Dentro de este gran campo se sitúa la radio universitaria, la cual debe entenderse como el resultado de un tipo de entramado relacional entre ciertos agentes -sujetos, sectores sociales, instituciones y el Estado- que establecen una lógica radiofónica específica, esto es, un subcampo dentro de una amplia gama de subcampos-radio educativa, radio indígena, indigenista, comunitaria, comercial, entre otras- que conforman el campo radiofónico. Es por lo anterior que saber la historia del campo radiofónico supone un ensamblado y reensamblado de procesos determinados que contribuyen a un conocimiento general del campo; sin embargo, el acercamiento a los subcampos contribuye a profundizar en su análisis para entender el estado actual de dicho subcampo. Es así que, dentro de las ciencias sociales, la radio debe entenderse como un proceso, más que como un objeto. El acercamiento a los subcampos radiofónicos y al entramado de relaciones de poder nos mostrará las posiciones de ventaja o desventaja que cada subcampo tiene dentro del campo radiofónico, y se verá que no es lo mismo la radio comercial respecto a la radio indígena o comunitaria, sobre todo frente al poder del Estado.

Otras nociones útiles para plantear este análisis son las que Bourdieu (2012) propuso sobre el capital. Según sus postulados, un individuo se sitúa en el espacio social de acuerdo con el volumen de capital económico, social, cultural y simbólico que posee. Los agentes -sujetos, sectores e instituciones- se mueven en el espacio social gracias a la acumulación de cada uno de éstos, y todos ellos son convertibles en otros tipos de capital. A continuación se delinea de forma breve a qué se hace referencia.

En cuanto al capital económico, Bourdieu señala que es convertible en dinero, y es una fuente esencial del poder político y la hegemonía (Bourdieu, 2010). En el campo radiofónico pueden observarse posiciones de ventaja, como en el caso de la radio comercial, la cual ha acumulado mayor capital económico mediante la publicidad principalmente, lo que le permite tener fuertes privilegios y mayor influencia dentro del campo radiofónico en México y el Estado, lo cual contrasta con (e implica un posicionamiento de poder de ventaja en relación con) la radio indígena o comunitaria. En cuanto al capital social, se asocian a él aquellos recursos inmateriales que se poseen por la pertenencia a determinado grupo, o bien por relaciones, redes de influencia y colaboración, de lo cual devienen, entonces, derechos, pero también obligaciones con los demás miembros del grupo con el que se está afiliado. Aquí opera la cercanía o distancia con los grupos mejor posicionados dentro del campo radiofónico (pues la cercanía facilita el acceso al ámbito, que puede reflejarse de distintas formas, ya sea en el acceso al trabajo remunerado o a la generación de contenidos a través de un espacio radiofónico, esto dependerá de cada caso específico o, por el contrario, siendo marginado del ámbito). Lo que constituye el capital cultural es la educación y las habilidades que tiene un agente 
social y que le otorgan un estatus determinado dentro de la sociedad, lo cual implica una formación que permite cierta movilidad a partir de la instrucción y el desarrollo de habilidades, así como su actualización en el conocimiento del medio, algunas radios analógicas se resisten a integrarse a las nuevas formas digitales de hacer radio, mientras que otras surgen a partir de esta incorporación tecnológica de la radio, lo que se refleja en posicionamientos de poder distintos dentro del campo radiofónico. Por último, se tiene al capital simbólico, que al igual que el social, se compone de propiedades intangibles inherentes al sujeto que únicamente pueden existir en la medida en que sean reconocidas por los demás. Su existencia depende del volumen de los otros tipos de capital y bien puede considerarse como el prestigio acumulado o poder adquirido por medio del reconocimiento de los agentes del campo. La formación de públicos puede identificarse como este capital simbólico, pues su generación, permanencia y crecimiento estarán en función del reconocimiento de sus públicos, a partir de un vínculo con determinado contenido y que, aunque no dependen de modo directo de los capitales anteriores, bien pueden contribuir para su fortalecimiento en el campo. El público de la radio comercial tendrá una composición, historia y expresiones diferentes al de una radio indígena o universitaria.

El capital cultural materializado no es capital cultural objetivo y simbólicamente dinámico, más que en el campo de fuerzas y luchas de producción cultural en la que los agentes usan su posición y su capital objetivado e incorporado. Debido a que su apropiación requiere de disposiciones y competencias que no son distribuidas de forma equitativa; las obras comerciales y culturales son objeto de apropiación exclusiva, tanto material como simbólica, usadas como capital cultural que consagra un beneficio de distinción y singularidad, lo cual se refleja en el consumo radiofónico (Bourdieu, 2012). En otras palabras, en el ámbito de las producciones radiofónicas, éstas son una materialización de capital cultural, el cual no es objetivo, sino simbólicamente dinámico, porque los agentes le aderezan a éste su posición y su propio capital. Por lo anterior, la apropiación y significación de las producciones radiofónicas se lleva a cabo de manera diferente de acuerdo con la suma global de los capitales. Por ende, un determinado capital cultural puede tomar rumbos muy distintos a los propósitos con los que en principio fue creado. En el campo de la radio se puede ver reflejado el valor que a los distintos contenidos o productos culturales se les otorga, pues no es igual el valor de la radio comercial frente a la radio pública, incluso hay diferencias dentro de la propia radio pública, que se valoran desde el tipo de lenguaje utilizado -contenidos radiofónicos educativos, contenidos musicales de experimentación, contenidos de revista o miscelánea o contenidos infantiles-, la función que cumplen, así como en los públicos que atrae. Lo que genera distancia y singularidad entre los agentes dentro del campo.

Lo que está claro es que el azar no rige el espacio social: hay fuerzas que imponen una estructura donde las clases oponen su propia inercia a través de sus propiedades. Tanto la posición como la trayectoria individual no son independientes, pues no existe la misma probabilidad entre los puntos de salida y los

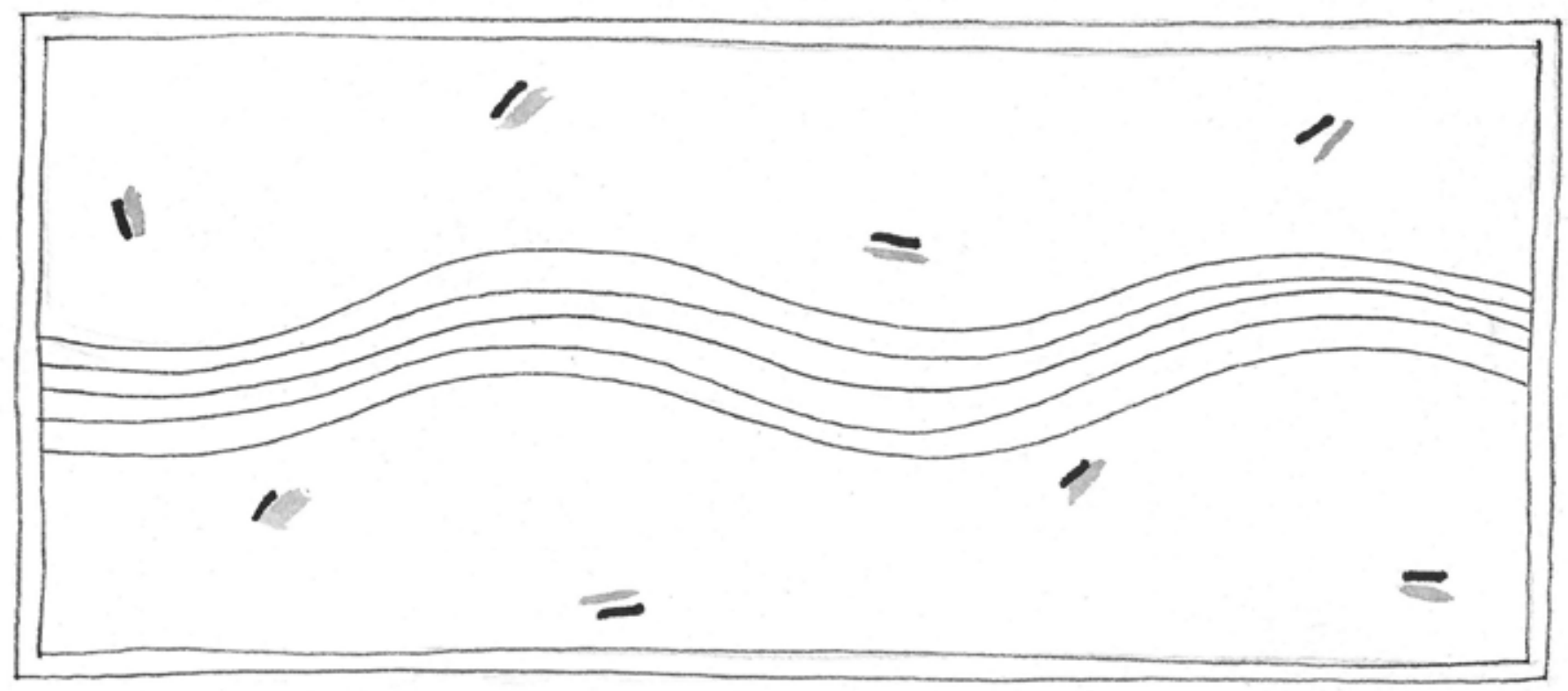


puntos de llegada (Bourdieu, 2012). Para Bourdieu "Es la lógica específica del campo, de lo que en él se encuentra en juego y de la especie de capital que se necesita para participar, lo que impone las propiedades mediante las cuales se establece la relación entre la clase y la práctica” (2012: 129). Así, el capital sería una especie de relación o energía social que sólo encuentra su valor dentro del campo donde se produce y reproduce. Sin embargo, en la práctica, las propiedades incorporadas o materializadas relacionadas con los agentes no resultan igualmente efectivas, es la lógica específica del campo y su mercado la que determina su valor como capital específico y, por consecuencia, como factor explicativo de las prácticas (Bourdieu, 2012). Entender esto permitirá saber que hay una lógica específica en el campo radiofónico, pues la estructura sobre la cual se estableció la radio pública en México está determinada por la fuerza que el Estado, como agente, otorgó a la radio comercial, lo que estableció las reglas del juego dentro del campo y una lógica donde algunos han logrado posicionarse mejor que otros. Así, la radio pública ha tenido que luchar para mantener su posición en la producción de bienes culturales a pesar de las desventajas de la lógica imperante.

Por lo dicho hasta el momento, el ámbito radiofónico debe concebirse como campo, esto permite entender su complejidad y diversidad, así como identificar a los agentes involucrados en su constitución y transformaciones a lo largo de la historia. A continuación se expondrá cómo, a partir de un panorama general, es posible comprender las fuerzas que han constituido al campo radiofónico en México.

\section{Conformación del campo radiofónico}

El campo radiofónico esconde una profunda complejidad que hasta el día de hoy no se ha podido agotar con los estudios existentes. Para acercarnos al imbricado laberinto lo haremos a través de este cuestionamiento: ¿cómo se ha conformado el campo radiofónico? A pesar de ser un mismo invento-objeto, los usos, los discursos, las apropiaciones y negociaciones, así como las posiciones de poder alrededor de la radio son diversas, y para algunos adversas, como para las radios comunitarias en relación con la comercial (Jocirin Auguste, 2006; Cebrián Herreros, 2001; Flichy, 1993). Es pertinente, desde ahora, diferenciar entre "el radio" como aparato físico, y "la radio" entendida como reflejo sociocultural del campo radiofónico. El concepto de institución mediática contribuye a identificar este proceso de conformación social en torno a la radio, pues son instituciones que crecen a partir de prácticas clave en la difusión de información y cultura, lo que implica una relación con otras instituciones (McQuail, 2001).

El final del siglo xIx y prácticamente todo el xx fueron años esenciales para el avance de la radio; y, del mismo modo que en varios países, en México el Estado se convirtió en uno de los agentes más importantes para su desarrollo, al ser el administrador y mediador de la práctica radiofónica, lo que posibilitó el paso de la radio originada como proyecto militar al político, hasta convertirse en proyecto de consumo (Cebrián, 2001; Rodríguez Montiel, 2010).

Hay diversas etapas políticas relacionadas con el desarrollo social de la radio, entre ellas están las de reconstrucción nacional por parte del Estado, mediante la cohesión social, propaganda política y educativa, la de bien de consumo doméstico y publicitario, como ordenador de prácticas sociales (Roldán Vera, 2009). Entre los años veinte y treinta, cuando México pasaba por una fase de reconstrucción nacional, se utilizó a la radio como vehículo de cohesión social posrevolucionaria. Por lo tanto, pronto pasó de ser un avance tecnológico a transformarse en un medio de unificación en el periodo posrevolucionario (en la década de 1930 aproximadamente). Ésta fue una de las formas más trascendentales para su expansión como herramienta de grupos políticos del Estado.

Llano y Morales (1984) sostienen que la radio ha sido el medio más económico para la integración de personas en el proceso de reconstrucción nacional por parte del Estado, la radio era de muy bajo costo de producción y de gran alcance, además, cubría un alto porcentaje de la sociedad, aspecto que podría reflejarse en su apresurada proliferación y asimilación cultural (Prieto de Ramos y Durante Rincón, 2007).

Pronto, la radio pasó de un uso político y militar a un pasatiempo personal y familiar debido al perfeccionamiento tecnológico que posibilitó dicho tránsito social. Esta práctica individual y grupal representó la incorporación al ámbito económico con transformaciones culturales significativas, como el hecho de que familias enteras desearan tener un radio, y que lo agregaran a su cotidianidad disponiendo de una cantidad de tiempo para ello. Este acelerado proceso fue tan relevante que hizo que cambiaran de manera conjunta tanto la misma producción de la radio como el consumo de los contenidos: la radio y la lógica del capital se encontraron. La radio se integró como un permanente compañero de las sociedades contemporáneas, que fue muy bien recibido por los distintos sectores sociales.

La radio transitó de un invento tecnológico a un ordenador de prácticas sociales, y contribuyó a la 
reorganización y administración del tiempo que la economía y la política imponían. También ayudó a que las sociedades se organizaran bajo un ritmo que la era industrial trazó, pasó del ámbito laboral y se filtró al doméstico, como lo señalan Lewis y Booth:

La radio, el primer medio de comunicación de masas, que giraba en torno al tiempo, impuso a sus oyentes los ritmos de la era industrial, y con la división del tiempo en minutos, implícitamente apoyó la noción de la precisión científica y la predictibilidad. En este sentido, la radio, según Scanell, cumple su primera tarea como una de las primeras organizaciones de la radiodifusión nacional: "la mediación de la modernidad"; con la introducción de las rutinas domésticas y la celebración de rituales nacionales para una comunidad de oyentes, no menos importantes por ser "imaginadas" en la mente de cada uno, el reciente medio de comunicación estaba intentando: "la normalización de la esfera pública y la socialización de la esfera privada" [Lewis y Booth, 1992: 92-93].

Las diferencias materiales entre los diversos sectores se hicieron presentes a través de la adquisición de ciertos aparatos, muy grandes y de madera fina para los sectores privilegiados, mientras que los sectores menos favorecidos económicamente los adquirían de dimensiones pequeñas y materiales sencillos. Esto trajo aparejado nuevas relaciones económicas de la radio como medio de comunicación y como práctica social y profesional, lo cual generó un nuevo campo de fuerzas bajo una nueva dinámica de relaciones (Jocirin Auguste, 2006; Prieto Ramos y Durante Rincón, 2007).

Se puede señalar entonces que la radio ha sido y es un campo en disputa, el cual ha sido apropiado por distintas estrategias y con diferentes finalidades por los múltiples sectores sociales. La práctica radiofónica está estrechamente relacionada con el contexto social, político, económico y cultural. Lo que convierte a la práctica radiofónica en un campo de poder con "espacios estructurados de posiciones", entendiendo esto como "un estado de relaciones de fuerzas entre los agentes o las instituciones que intervienen en la lucha" (Bourdieu, 2002: 120), posiciones que se han transformado en las diferentes épocas y contextos, pero donde el Estado ha sido el principal agente de tensión. En palabras de Bourdieu:

Un campo [...] se define, entre otras formas, definiendo aquello que está en juego y los intereses específicos, que son irreductibles a lo que se encuentra en juego y a sus intereses propios $[\ldots]$ y que no percibirá alguien que no haya sido construido para entrar en ese campo [...] Para que funcione el campo es necesario que haya algo en juego y gente dispuesta a jugar [Bourdieu, 2002: 119-120].

Esto supone que el campo radiofónico está integrado por subcampos que, si bien tienen diferencias específicas, comparten un espacio político, social, económico y cultural, por lo que ocupan distintos lugares respecto al poder, pero al mismo tiempo están interrelacionados, lo que define su condición de posibilidad de hacer radio, reflejo de la estructura social en México dependiendo de la cercanía e interés del Estado.

\section{La radio en México ${ }^{3}$}

El Estado jugó un papel fundamental en el establecimiento y desarrollo de la radio en México, ya que de forma paralela a la radio comercial se desplegó un proyecto educativo nacional basado en el medio radiofónico. Por consiguiente, podría decirse que la radio en México tiene influencia de la radio europea, en cuanto a la existencia de una radio pública, pero también de la estadounidense, sobre todo en el modo en que se ha extendido la radio comercial. Hasta hoy, el modelo estadounidense de la radio comercial ha mantenido mayor influencia entre la población mexicana. La radio comercial, que tiene como principal fuente de financiamiento los formatos básicos de la publicidad, contrasta con el modelo europeo que posee un gran servicio radiofónico público. La observación que hace la investigadora Ana María Peppino Barale (1999) es que, mientras el modelo público invierte en educar a las audiencias, el privado vende su audiencia a los publicistas.

La radio en México está regulada por el Estado, es el agente encargado del otorgamiento legal del uso de la ondas hertzianas; por mucho tiempo existió la figura de concesión -exclusivamente para uso comercial-y permiso -para un uso no comercial-, esta condición no permitió el acceso legal a iniciativas independientes -sectores indígenas, rurales, urbanos, comunitarios, entre muchos otros-, por lo que ha habido escenarios distintos para los sectores con menores recursos económicos y políticos, es decir, los mejor posicionados en el campo radiofónico lograron obtener concesiones para operar una frecuencia de tipo comercial

\footnotetext{
3 Merece la pena mencionar que los subcampos no existen aislados, están en constante interrelación y esto genera influencia mutua, aquí se presentan de manera descriptiva.
} 
a diferencia de los sectores que pretendían aspirar a tener una radio permisionada ${ }^{4}$ (OSF, 2011) sin fines de lucro.

El Estado mexicano en su papel de regulador del espectro radioeléctrico establece reglas en su acceso, frente a esto, algunos grupos quedan excluidos, por lo que buscan maneras de utilizar dicho espectro para su aprovechamiento y con ello el logro de distintos objetivos colectivos, a esto se le llamó en algún momento radio pirata. Sin embargo, el significado que diversos grupos le dan al uso de la radio puede ayudar a mantener aspectos fundamentales como la circulación de valores compartidos, expresión de la identidad, difusión de conocimiento u organización social, entre otros, lo que puede fortalecer vínculos entre quienes generan contenidos radiofónicos y sus públicos, como es el caso de las radios comunitarias e indígenas, entre otros. A continuación se mencionan algunas características concretas de las radios que operan en México, ${ }^{5}$ se inicia con la radio comercial, la mejor posicionada frente al Estado, después la radio indígena e indigenista, luego la comunitaria y por último la universitaria. ${ }^{6}$

\section{Radio comercial}

En 1930, la estación XEW dejó atrás la radio experimental de principios de siglo y abrió paso a la radio comercial. En este proceso ya destacaban tres agentes relevantes: los empresarios en búsqueda de aumentar sus ganancias; el Estado -a través del gobierno-, que trataba de generar su propaganda, así como de difundir educación y la cultura a su población; y los radioescuchas, con expectativas de información y entretenimiento. Es importante recordar el pacto establecido entre esta empresa y el Estado, a través del apoyo financiero y tecnológico a cambio de difusión ideológica y censura (Rodríguez Montiel, 2011). A partir de lo anterior se logró el desarrollo de una gran infraestructura, cuyo resultado fue la rápida propagación y difusión de los aparatos radiofónicos y con ello los contenidos comerciales en México (Sosa Plata, 2008 y 2016).

Los orígenes de la industria de la radio en México están estrechamente vinculados a la relación entre el Estado y las élites empresariales, así como a grupos económicos asociados a capital extranjero, lo que impulsó su desarrollo comercial. Según Mejía Barquera (1991) entre 1920 y 1928 se generaron las condiciones políticas y jurídicas para regularizar su funcionamiento comercial con claros límites de acción estatal, para que los capitales extranjeros y nacionales pudieran invertir de manera segura. A partir de entonces, sobrevino un crecimiento acelerado de la industria radiofónica comercial en México, que fue la que estableció el modelo y los lineamientos para la evolución de la industria nacional de la radio comercial en México (Ortega Ramírez, 2005). Esta radio ha gozado de las mayores ventajas respecto a otros modos de hacer radio, ocupa la posición más ventajosa por su estrecha relación con el Estado y por su acumulación de capital, que le permite generar otro tipo de capitales. La producción de conocimiento sobre esta radio es principalmente técnica, además de estar muy vinculada con la formación de profesionistas mediante las carreras en comunicación en México y ha sido la de mayor influencia en los públicos radiofónicos mexicanos.

\section{Radio indigenista e indígena}

Las radios indígenas e indigenistas son dos proyectos distintos. La radio indigenista surge a partir de un enfoque político desarrollista por parte del Estado, con base en la idea de que la radio sería utilizada como herramienta de alfabetización y castellanización, para incorporar a los grupos indígenas en los programas de desarrollo rural del gobierno mediante el Instituto Nacional Indigenista (INI) ${ }^{7}$ a través del Sistema de Radiodifusión Cultural Indigenista (Cornejo Portugal, 2010). Gasparello (2011) sostiene que la primera estación se instaló en 1979 en Tlapa, Guerrero, su intención era ser un canal de apoyo para las labores gubernamentales, operadas sobre todo por élites vinculadas al gobierno, las cuales, al monopolizar la información, lograron reducir la pluralidad de voces y controlar zonas indígenas.

Por otra parte, existe la radio indígena (distinta de la indigenista). Algunos de los elementos que la cons-

4 De 2006 a 2015 el gobierno no otorgó ninguna licencia de radiodifusión e ignoró 140 solicitudes.

5 La mayor producción literaria sobre la radio en México se genera a partir de las radios comercial, comunitaria, indígena e indigenista y universitaria. Con este criterio es que sólo se hace mención de algunas, faltando las religiosas, las alternativas, las de guerrilla, las piratas, entre un sinfín más.

6 Hay pocos trabajos que aborden la radio universitaria, entre ellos está la investigación mía con Zael Ortega Pérez (Güemes Cruz y Ortega Pérez, 2017), donde reconstruimos la historia de lucha del sector universitario frente al Estado mexicano por la obtención del permiso para operar la Radio Universitaria de la Benemérita Universidad Autónoma de Puebla (BUAP).

7 En 2003, el INI fue sustituido por la Comisión Nacional para el Desarrollo de los Pueblos Indígenas (CDI) (Diario Oficial de la Federación, 21 de mayo del 2003). 
tituyen, según Castells I Talens (2011), son: un perfil en común, que no son comerciales, transmiten de manera cotidiana en lenguas indígenas y también en español, sus funciones se inspiran en lógicas comunitarias y cuentan con gran popularidad entre su población. Ginsburg (en Gasparello, 2011) advierte sobre tres condiciones que permitieron su surgimiento: la búsqueda de autonomía cultural y de la tierra a partir de luchas indígenas; la descentralización de las tecnologías, y la existencia de políticas multiculturalistas con formas concretas de establecer derechos políticos y culturales de los grupos indígenas. Este modo de hacer radio comparte algunos principios de la radio comunitaria, sin embargo, se plantea y atiende problemáticas de comunidades indígenas locales, hace uso de lenguas indígenas y, por lo tanto, genera una circulación de significados culturales específicos con los que se identifican sus oyentes. La radio indígena no depende del gobierno, pues implica una postura de tensión sobre la condición política de los pueblos indígenas con el Estado a través de la radiofonía.

En los años noventa se conocieron algunos de sus problemas y limitaciones, pero también quedó claro que se cimentaba como instrumento de comunicación por el que circulaban recursos simbólicos (música, lengua, noticias de las localidades) y otros aspectos que algunos grupos indígenas reconocieron y rápidamente replantearon (Cornejo Portugal, 2010). Diversos movimientos sociales indígenas visibilizan a sus públicos como sujetos activos con capacidad de organización y transformación de su entorno y no como un receptor que se limita al acto de escuchar o consumir; las radios indígenas se volvieron más poderosas y, por consecuencia, peligrosas para el Estado (por la autogestión y control de sus propias radios). Por este motivo, no es fácil que se les otorguen permisos (ahora concesiones sociales).

El modelo de la radio indigenista, en los años ochenta, tenía el objetivo de rescatar, conservar y difundir la cultura indígena, pero se ha observado que la radio indígena ha contribuido con una nueva propuesta como modelo radiofónico, ya que, además de incorporar organizaciones productivas y culturales indígenas en su producción radiofónica, "constituye el medio más importante de comunicación para los pueblos indios" (Cornejo Portugal, 2010: 56).

En muchos casos, los proyectos gubernamentales de la radio indigenista fomentaron la apropiación de la radio por parte de grupos indígenas debido a dos características fundamentales; por un lado, trabajan indígenas de las propias comunidades y, por el otro, se inicia un formato que no tenía antecedentes en América Latina.

\section{Radio comunitaria}

Las radios comunitarias surgen como propuestas alternativas a través de proyectos en territorios locales, que por lo regular se articulan a redes más amplias de organización. Existe una vinculación entre organización social y medios de comunicación, su objetivo puede ser evidenciar las asimetrías, las injusticias, así como las viejas y nuevas formas de dominación (Gerbaldo, 2008).

Gerbaldo sostiene que: "podemos señalar que, buena parte de lo que los sujetos y las personas viven, se afinca en la relación que las nuevas tecnologías y los medios de comunicación contemporáneos instalan con otras formas de socialidad [...] disponiendo ciertos hablantes, y ciertos espacios comunicacionales, desde donde se construyen identidades diversas" (Gerbaldo, 2008: 4). La autora señala que el término radio comunitaria se usa en la actualidad en remplazo de radio popular, que, como categoría, cayó en desuso por aludir de manera implícita a la condición de clase. Para Aleida Calleja y Beatriz Solís (2007) tiene una estrecha relación con la categoría de ciudadanía, por lo que su interés también incluyó la defensa de la democracia y problemas concretos de comunidades específicas.

La defensa de los derechos humanos, la promoción de un desarrollo humano sostenible, la equidad de género, el respeto a las identidades étnicas, la preservación del

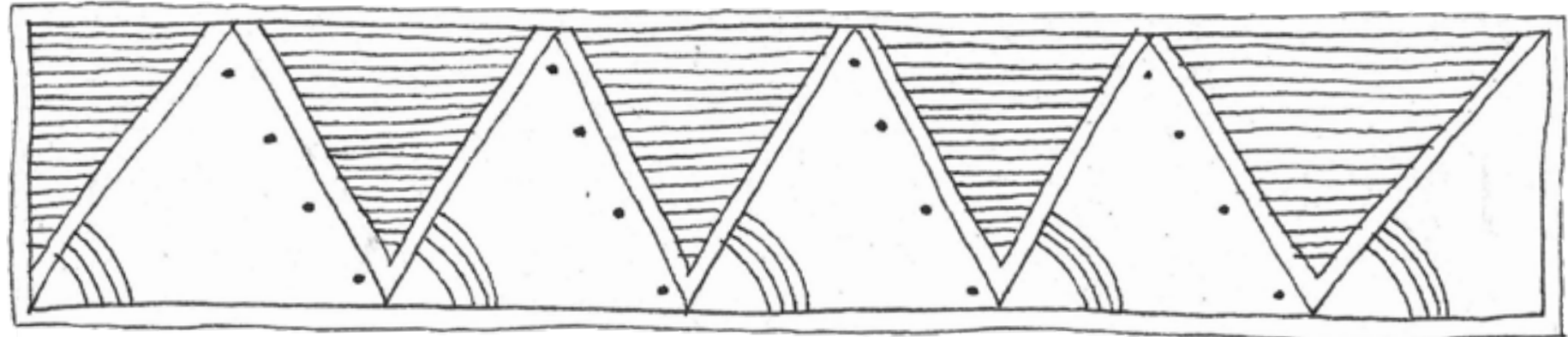


medio ambiente, el protagonismo de los jóvenes y sus propuestas, la protección a la niñez y de las personas adultas mayores, la educación y la salud, así como la integración nacional y regional, constituyen los ejes fundamentales que determinan el perfil de las radios comunitarias [Calleja y Solís, 2007: 23].

Como todo subcampo radiofónico, la radio comunitaria no queda exenta de conflictos, diferencias, ni tampoco de tensiones con el Estado o con el mercado, por el contrario, se ven atravesadas por éstas, y los agentes que crean y generan estos proyectos radiofónicos buscan mantenerse a pesar del contexto en el cual están inmersos. Todo esto produce una lógica diferente respecto de la lógica de la radio comercial, pues en la radio comunitaria lo esencial es la comunidad, la organización y sus integrantes; su finalidad es social, siempre dentro de una esfera no lucrativa (Gerbaldo, 2008).

Para otros autores "la especificidad de las radios comunitarias, tiene que ver esencialmente con el proceso organizativo que está detrás de la radio y con los vínculos que ésta establece con la comunidad en la que transmite" (Gasparello, 2011: 29). Entre sus objetivos está que cumplan una función social a través de la transformación social para el beneficio de su comunidad mediante contenidos que induzcan su participación en aras de la reflexión, la educación y la difusión de su propia cultura. Por lo anterior puede entenderse a la radio comunitaria como una forma política de participación social.

La radio comunitaria y ciudadana encuentra su definición en el ejercicio de los derechos ciudadanos, pretende reconstruir el tejido social a partir de la diferencia y la democracia, a través del uso de derechos políticos, civiles y sociales que conduzcan a una vida digna (individual y colectiva), como sintetizan Calleja y Solís:

En este sentido, una radio comunitaria o ciudadana, es una propuesta social que un colectivo u organización ofrece a la sociedad, exponiendo claramente cómo pretenden estar y en consecuencia incidir en ese tejido social. Entendida así, la radio comunitaria es un proyecto político, en el sentido de que asume compromisos y toma posición respecto a la problemática concreta en que vive y se ubica en el contexto social en donde se desarrolla [Calleja y Solís, 2007: 24].
Para la Asociación Mundial de Radios Comunitarias (AMARC) ${ }^{8}$ el objetivo de las radios comunitarias es que la comunidad haga algo por ella misma, pues la comunidad es su lugar de reconocimiento y forma de participación.

\section{Radio universitaria}

De acuerdo con la Ley Federal de Radio y Televisión, las radios universitarias persiguen fines académicos a partir de la difusión de la cultura y el conocimiento con un propósito educativo. La radiodifusión universitaria tendría que ser una expresión de la cultura y el conocimiento científico producidos en las instituciones de nivel superior (Pérez Rosas, 2004), es por ello que está muy relacionada con la radio educativa y cultural, pero con la diferencia de que pertenece a un sector específico: el universitario. No obstante, no hay una distinción clara y formal entre la radio educativa, la cultural y la universitaria, de ahí que la sugerente propuesta de Martín-Pena, Parejo Cuéllar y Vivas Moreno (2016) de asumir a la radio universitaria como un elemento más dentro de un sistema complejo de información sea una tarea pendiente.

En 1937 surge la primera radio universitaria en México llamada Radio UNAM, la emisora de la Universidad Nacional Autónoma de México. Fue la primera estación que marcó el modelo de radio universitaria y que hasta hoy sigue teniendo influencia nacional en los proyectos radiofónicos de este tipo (Marta-Lazo y Martín-Pena, 2014).

Existen múltiples definiciones de lo que caracteriza a la radio universitaria, sin embargo, considero que no es fácil establecer una definición actualizada, es decir, que se ajuste a las transformaciones sociales contemporáneas sobre el papel de la radio universitaria, por lo que es necesario estudiar más sobre su trabajo y así poder profundizar en el conocimiento de su quehacer actual. Si entendemos que en la universidad se realizan, por lo menos, tres actividades de suma trascendencia para la sociedad, como lo son la docencia, la investigación y la difusión de la cultura, así como la extensión universitaria, tendríamos un punto de partida para entender los objetivos de la radio universitaria. Es así que, dentro de las labores de la difusión cultural y extensión universitaria, bien se podrían compartir los

8 La AMARC, como se define en su página web (www.amarc.org): "es una organización no gubernamental internacional al servicio del movimiento de la radio comunitaria, que agrupa cerca de 4000 miembros y asociados en más de 110 países. $\mathrm{Su}$ objetivo es apoyar y contribuir al desarrollo de la radio comunitaria y participativa de acuerdo con los principios de solidaridad y la cooperación internacional. En el Consejo Internacional de AMARC se encuentran representados todos los continentes". 
avances en materia de investigación, innovaciones, discusiones, críticas y reflexiones tanto humanísticas como tecnológicas para la transformación de la realidad, sin caer en la dinámica de una clase universitaria (Pérez Rosas, 2004).

Pérez Rosas (2004) cree importante distinguir dos cuestiones sobre las radios universitarias. La primera comprende el uso de la radio dentro de las universidades y la segunda es la radiodifusión como tal. A esto yo añadiría la incorporación de las nuevas tecnologías de la información, porque reconfiguran su uso (Marta-Lazo y Martín-Pena, 2014; Martín-Pena, Parejo Cuéllar y Vivas Moreno, 2016). De la primera cuestión, la radio es utilizada para atender necesidades comunicativas en el interior de la institución y, en este caso, no se cuenta con la plataforma institucional o la infraestructura de una radio formal, y se parecería más a un circuito cerrado y no a la radiodifusión universitaria, pues está dirigida a la formación estudiantil y no al consumo de sus propuestas culturales. La segunda es la radiodifusión y para ser considerada como universitaria debe contar con los siguientes aspectos: ser de carácter masivo, transmitir desde un recinto universitario y bajo una frecuencia dentro del cuadrante (AM O FM), además de tener un transmisor y una antena, así como el respaldo de una institución de educación superior (IEs). Estas peculiaridades diferencian a la radio universitaria de otras formas de hacer radio dentro de las universidades $y$, por ende, no puede llamarse radio universitaria a cualquier proyecto de radio. Pese a ello, las universidades han buscado formas de difundir su trabajo y combinan maneras de hacerlo. Si bien no todas cuentan con los elementos mencionados, esto no significa que no realicen trabajo de difusión de su labor. En la actualidad, las innovaciones tecnológicas han replanteado las modalidades tradicionales de hacer radio universitaria, el internet ha sido una herramienta que permite a las universidades desarrollar habilidades en sus estudiantes para la práctica profesional. Todo esto nos obliga a cuestionarnos qué es lo que define a la radio universitaria: sus condiciones materiales o su labor radiofónica a pesar de no tener una frecuencia en el cuadrante. Es menester generar nuevas preguntas sobre este subcampo radiofónico, pues debe ser más explorado y conocido.

\section{Reflexión final}

Este trabajo sostiene que el concepto de campo de Bourdieu -entendido como espacio de lucha, tensión y apropiación simbólica-, aplicado a la radio, permite re- velar la complejidad del fenómeno radiofónico, en particular en México. Uno de los aportes de este concepto es que demanda conocimiento sobre los diversos subcampos, en este caso de las diversas formas de hacer radio en el país. Develar su complejidad implica analizar con detalle las tensiones y posicionamientos que cada subcampo radiofónico enfrenta en relación con el poder. Sin embargo, provee un acercamiento general al entramado de relaciones de poder que, como se verifica en México, se sustentó a partir de desigualdades, al tiempo que también generó potencialidades en cada subcampo radiofónico. Diversos son los estudios que han revelado sus características, problemáticas y aportes. Parece imperativo conocer la situación actual de campo radiofónico en México, sobre todo a partir de las nuevas condiciones estructurales del país y su impacto en la práctica radiofónica cotidiana, porque hacer radio en este país no ha significado lo mismo para todos los sectores sociales, su acceso es el reflejo de una estructura social desigual que durante muchos años ha existido, con privilegios para un subcampo específico, como la radio comercial, que estableció pautas generales en su práctica. El aporte de esta categoría de campo de poder conlleva iniciar el acercamiento al fenómeno; en cuanto herramienta de análisis se espera que las nuevas modificaciones en materia de comunicaciones generen las condiciones para un acceso más justo de los diversos sectores de la sociedad que han encontrado históricamente en la radio un espacio de lucha y apropiación simbólica y material, así como de interacción social, y con ello la restauración del tejido social en México. La práctica radiofónica es y seguirá siendo una práctica en disputa, no obstante, las transformaciones tecnológicas han reconfigurado tanto las relaciones de poder, como las tensiones y las luchas dentro del campo radiofónico, por lo que debe ser examinado a partir de estas nuevas configuraciones, es así que se afirma que la categoría de campo radiofónico -a partir de Bourdieu-es vigente para analizar el fenómeno radiofónico en México y la tarea pendiente es profundizar en la interrelación de los campos y su mutua influencia.

\section{Fuentes}

Bourdieu, Pierre

2002 Campo de poder, campo intelectual. Itinerario Bourdieu, Pierre de un concepto, Montressor, Buenos Aires.

2010 "Consumo cultural", en Pierre Bourdieu, El sentido social del gusto. Elementos para una sociología de la cultura, Siglo xxi Editores, Buenos Aires, pp. 231-240. 
Bourdieu, Pierre

2012 La distinción. Criterios y bases sociales del gusto, Taurus, México.

Calleja, Aleida

Y BEATRIZ SOLÍS

2007 Con permiso. La radio comunitaria en México, Asociación Mundial de Radios ComunitariasMéxico/Asociación Mexicana del Derecho a la Información/Comunicación Comunitaria/ Comisión Mexicana de Derechos y Promoción de los Derechos Humanos, México.

Castells I Talens, Antoni

2011 ¿Ni indígena ni comunitaria? La radio indigenista en tiempos neoindigenistas", en Sociedad y Comunicación, núm. 15, enero-junio, pp. 123-142.

Cebrián Herreros, Mariano

2001 La radio en la convergencia multimedia, Gedisa, Barcelona.

Cornejo Portugal, Inés

2010 "La radio cultural indigenista en México: dilemas actuales", en Revista Mexicana de Ciencias Políticas y Sociales, vol. LII, núm. 209, mayo-agosto, pp. 55-66.

Flichy, PATRICE

1993 Una historia de la comunicación moderna, Ediciones G. Gili, México.

Gasparello, Giovanna

2011 "Donde crece la flor de la palabra. Reflexiones sobre la radio comunitaria indígena en los estados de Guerrero y Oaxaca, México", en Nómadas, Critical Journal of Social and Juridical Sciences, vol. 29, núm. 1, enero-junio.

Gerbaldo, Judith

2008 "Hacia una cartografía de las radios comunitarias argentinas", ponencia presentada en $10^{\circ}$ Congreso Redcom "Conectados, Hipersegmentados y Desinformados en la Era de la Globalización", septiembre, Universidad Católica de Salta-Facultad de Artes y Ciencias.

Güemes Cruz, Anayuri

2017 "El campo radiofónico universitario, sus agentes y sus públicos: caso de Radio BUAP", tesis doctoral, Universidad Autónoma Metropolitana-Iztapalapa, México.

Güemes Cruz, Anayuri

y Zael Ortega Pérez

2017 "La historia de una radio negada. Radio UAP: La voz de la justicia y la razón (1937-1997)", en El periodismo digital y las nuevas configuraciones del lenguaje, Agustín René Solano Andrade, Israel León O'Farril y Andrea Estupiñán Villanueva (coords.), El Errante Editor/ Benemérita Universidad Autónoma de Puebla, México, pp. 163-187.

Jocirin Auguste, Patricia

2006 "La radio en la ciudad de México", tesina de licenciatura en Historia, Universidad Autónoma Metropolitana-Iztapalapa, México.

LATOUR, BRUNO

2008 Reensamblar lo social. Una introducción a la teoría del actor-red, Manantial, Buenos Aires.

Lewis, Peter

Y JERRY BOOTH

1992 El medio invisible. Radio pública, privada, comercial y comunitaria, Hurope, Barcelona.

Llano, Serafina y Óscar Morales

1984 Laradiodifusiónen México, Comunicación:Tecnología e Investigación, México.
Marta-Lazo, Carmen

y DANiEl Martín Pena

2014 "Investigación sobre radio universitaria: Presente, pasado y futuro", en Revista de Educación Mediática y TIC, vol. 3, núm. 1, pp. 8-25.

Martín-Pena, Daniel, Macarena Parejo Cuéllar

y Agustín Vivas Moreno

2016 La radio universitaria. Gestión de la información, análisis y modelos de organización, Gedisa, Barcelona.

McQuaIL, Denis

1998 La acción de los medios. Los medios de comunicación y el interés público, Amorrortu, Buenos Aires.

McQuail, Denis

2001 Introducción a la teoría de comunicación de masas, Paidós, México.

Mejía Barguera, Fernando

1991 La industria de la radio y televisión y la política del Estado mexicano (1920-1960), vol. I, Fundación Manuel Buen Día, México.

Ortega Ramírez, Carmen Patricia

2005 "Medios públicos en el Estado mexicano moderno", tesis de doctorado en Ciencias Políticas y Sociales con orientación en Ciencias de la Comunicación, Universidad Nacional Autónoma de México.

OSF

2011 Open Society Fundation. Los Medios digitales en México.

Peppino Barale, Ana María

1999 Radio educativa, popular y comunitaria en América Latina: origen, evolución y perspectivas, Plaza y Valdés, México.

Pérez Rosas, Luis David

2004 "La radiodifusión universitaria y los circuitos cerrados de radio en las instituciones de educación superior: una propuesta para la expresión de los estudiantes", en Revista Mexicana de Ciencias Políticas y Sociales, vol. 47, núm. 192, septiembre-diciembre, pp. 153-186.

PRIETO DE RAMOS, IRIS

Y Esther DuRANTE Rincón

2007 "La evolución de la radio y las implicaciones tecno-socio-culturales en la audiencia: de oyente a usuario en la recepción del mensaje", en Espacio Abierto. Cuaderno Venezolano de Sociología, vol. 16, núm. 2, abril-junio, pp. 313-329.

Rodríguez Montiel, Elizabeth

2010 "El fenómeno histórico de la radio en México", en Razón y palabra, núm. 69, Deporte, cultura y comunicación, julio-septiembre, pp. 1-8.

Rodríguez Montiel, Elizabeth

2011 "Nativos digitales en la reconfiguración radiofónica”, en Razón y Palabra, núm. 76, mayojulio.

Roldán Vera, Eugenia

2009 "Los orígenes de la radio educativa en México y Alemania: 1924-1935", en Revista Mexicana de Investigación Educativa, vol. 14, núm. 40, pp. 13-41.

Sosa Plata, Gabriel (COORD.)

2008 Radio Educación. La historia reciente, testimonios y remembranzas, Secretaría de Educación Pública/Radio Educación, México.

Sosa Plata, Gabriel (COORD.)

2016 Días de Radio. Historias de la radio en México, Secretaría de Cultura, México. 